\title{
Stem cell markers (cytokeratin 15, cytokeratin 19 and p63) in in situ and invasive cutaneous epithelial lesions
}

\author{
Ossama Abbas, Joanna E Richards, Ron Yaar and Meera Mahalingam \\ Dermatopathology Section, Department of Dermatology, Boston University School of Medicine, \\ Boston, MA, USA
}

\begin{abstract}
The inherent longetivity of stem cells causes them to be susceptible to multiple genetic hits. Thus, it is not surprising that stem cells are implicated in the etiopathogenesis of select cutaneous neoplasms. However, most studies to date are restricted to the use of a single marker (p63, cytokeratin-15 or cytokeratin-19) and do not appear to compare distribution of stem cell markers in a spectrum of cutaneous in situ versus invasive epithelial malignancies. In this study, we evaluate expression of cytokeratin-15, cytokeratin-19, and p63 in a series of primary cutaneous epithelial lesions that include actinic keratosis $(n=29)$, squamous cell carcinoma in situ $(n=30)$, bowenoid papulosis $(n=15)$ and squamous cell carcinoma, well differentiated $(n=29)$ in order to evaluate the role of stem cell marker expression in the grading and development of in situ and invasive malignancies. For cytokeratin-15, expression was retained in actinic keratosis $(38 \%)$, squamous cell carcinoma in situ $(53 \%)$ and bowenoid papulosis $(60 \%)$ but appeared to be lost in squamous cell carcinoma $(3 \%)$ with statistically significant differences observed between groups that retained versus those that did not $(P<0.05$ for all three); for cytokeratin-19, patchy yet basal expression was noted in actinic keratosis (21\%), patchy and suprabasal expression was noted in squamous cell carcinoma in situ (37\%), bowenoid papulosis (13\%) and squamous cell carcinoma $(24 \%)$ with no statistically significant differences between groups; for p63, expression was retained in actinic keratosis $(90 \%)$, squamous cell carcinoma in situ (87\%), bowenoid papulosis $(60 \%)$ and squamous cell carcinoma $(100 \%)$ with no statistically significant differences between groups. In summary, our findings expand the neoplasms which involve stem cells to include cutaneous epithelial malignancies. Differential localization of each of these markers argues in favor of stem cell heterogeneity. Modern Pathology (2011) 24, 90-97; doi:10.1038/modpathol.2010.180; published online 17 September 2010
\end{abstract}

Keywords: actinic keratosis; bowenoid papulosis; CK15; CK19; p63; squamous cell carcinoma; squamous cell carcinoma in situ

Stem cells in human skin are adult or tissue-specific stem cells that maintain normal tissue turnover and repair by replenishing specialized differentiated cells. ${ }^{1}$ Their main characteristics include the ability to renew indefinitely (self-renewal) and multipotency or the capacity to differentiate into multiple specialized cell lineages of the specific tissue., ${ }^{1,2}$ Given that the existence of varied populations of stem cells in human skin with diverse differentiation

Correspondence: Dr M Mahalingam, MD, PhD, FRCPath Dermatopathology Section, Department of Dermatology, Boston University School of Medicine, 609 Albany Street, J-301, Boston, MA 02118, USA,

E-mail : mmahalin@bu.edu

Received 13 April 2010; revised 21 July 2010; accepted 28 July 2010; published online 17 September 2010 capacities is now indisputable, more recent evidence implicating stem cells in the etiopathogenesis of cutaneous neoplasms is not entirely surprising. ${ }^{3-5}$ Properties of self-renewal, increased profilerative capacity and multipotency, inherent to a normal stem cell, are believed to be of relevance in tumor maintenance. ${ }^{1,3-5}$ There are some who believe that, in select cancers, the lesional neoplastic cells may actually originate from mutated normal stem cells., ${ }^{1,3-5}$

Studies first suggested cytokeratin-15 (CK15) as a specific marker of stem cells when a restricted CK15-expression was demonstrated in cells of the bulge region of murine and human hair follicles. ${ }^{1,6,7}$ However, our own and other studies have shown that CK15 expression is not only restricted to the bulge, but also involves part or all of the outermost layer of the outer root sheath of the human hair 
follicle, the basal layer of the epidermis, and even eccrine glands. . $^{1,2,8}$ Designation of CK15 as a stem cell marker is based on evidence indicating that CK15 positive cells in the bulge region and interfollicular epidermis possess characteristics of stem cell such as self-renewal and multipotency. ${ }^{1,6,7}$ Expression of CK15 in the mitotically active basal cell layers of the hair follicle suggests that CK15 may have a role in regulating an early stage of keratinocytes differentiation and one that predates the fate of a cell becoming epidermal or hair-like. ${ }^{1,2}$

Similar to expression of CK15, expression of cytokeratin-19 (CK19) was first thought to be restricted to the bulge region of the hair follicle, but more recent studies have shown up-regulated expression of CK19 in the outermost layer of the outer root sheath in the bulge region of the human hair follicle as well as the outer root sheath proximal and distal to the bulge. ${ }^{1,2,10,11}$ In vivo and in vitro studies have shown that CK19 may be important in the commitment of stem cells to an epidermal cell fate and differentiation..$^{1,2,12}$

Transcription factor p63 is considered to be a homologue of the p53 tumor suppressor gene. ${ }^{13-15}$ Although in vitro studies have initially identified p63 as a keratinocyte stem cell marker, more recent in vivo studies have shown that p63 expression is not restricted to epidermal stem cells but also involves basal and suprabasal epidermal cells as well as the outer root sheath and hair matrix of hair follicles. ${ }^{13-15}$ Thus, it may be a marker of transient amplifying cells rather than of stem cells. Compared to stem cells, transient amplifying cells are more differentiated and committed unipotent cells that have lost the ability to self-renew and can only go through limited rounds of proliferation before undergoing terminal differentiation. ${ }^{1}$

Despite the promiscuous distribution of CK15, CK19 and p63, these markers are still being used as stem cell markers, based on irrefutable evidence that a proportion of the cells in the skin that stain positively with these markers have stem cell characteristics of self-renewal and multipotency. ${ }^{1,2,6,7,10,11,13-15}$ In this study, we perform immunohistochemical stains to CK15, CK19, and p63 in a series of primary cutaneous epithelial lesions that include actinic keratosis, squamous cell carcinoma in situ, bowenoid papulosis, and squamous cell carcinoma in order to determine the following:

1. Is there a significant presence of stem or progenitor cells in these entities?

2. Is the population homogenous?

3. Do stem cells play a role in the grading and development of in situ versus invasive malignancies?

\section{Materials and methods}

The study was approved by the Institutional Review Board of Boston Medical Center (H-28797). Archival materials with a diagnosis of actinic keratosis, squamous cell carcinoma in situ, squamous cell carcinoma and bowenoid papulosis were retrieved from the data base of the Skin Pathology Laboratory, Boston University School of Medicine, Boston, MA. A total of one hundred and three cases (30 cases of squamous cell carcinoma in situ, 29 of actinic keratosis, 29 of well differentiated squamous cell carcinoma and 15 of bowenoid papulosis) fit criteria for inclusion in the study. Cases of bowenoid papulosis cases were selected on the basis of clinicopathological correlation and not based on histology alone. The histologic sections of all cases were re-reviewed and the diagnoses confirmed by the dermatopathologist (MM). Clinical information was extracted from the medical records. All patient data were de-identified.

\section{Immunohistochemical Analysis}

Five-micrometer-thick sections were obtained for immunohistochemical studies, which were performed on formalin-fixed, paraffin-embedded tissue using standard peroxidase immunohistochemistry techniques, heat-induced epitope retrieval buffer and primary antibodies against CK15 (LHK15; 1:250, NeoMarkers, Fremont, CA), CK 19 (MO888; 1:100; Dako, CA, USA) and p63 (7JUL, 1:25, NovoCastra, Newcastle upon Tyne, UK). Appropriate positive and negative controls were included. All stained slides were initially reviewed and scored by the first author (OA) and re-reviewed by the dermatopathologist (MM) in a blinded fashion to ensure consistency of interpretation.

Positive CK15 staining was noted by ascertaining cytoplasmic expression. Any nuclear staining was considered background artifact. In each case, CK15 was expressed in the basal layer of the epidermis, the outer root sheath of the hair follicle and secretory cells of eccrine glands and this served as positive internal control. As CK15 is normally expressed in the basal cell layer of the normal epidermis, CK15 expression was evaluated in the lesion in comparison to adjacent normal epidermis in each of the categories. Expression of CK15 in the cytoplasm of lesional cells was scored as $0(<25 \%$ of positively-staining tumor cells), $1+(25-75 \%$ of positively-staining tumor cells), and $2+(>75 \%$ of positively-staining tumor cells).

Positive CK19 staining was noted also by ascertaining cytoplasmic expression. Any nuclear staining was considered background artifact. In each case, CK19 was expressed in the bulge area of the hair follicle, the outer root sheath of the follicle just above and below the bulge area, and eccrine glands and this served as positive internal control. Cytokeratin-19 is not normally expressed in the normal epidermis. Thus, CK19 expression was evaluated in lesions exhibiting the same. Overall, positive CK19 expression tended to be patchy, staining a cluster or 
aggregate of cells and not diffuse. Positive CK19 expression in the cytoplasm of lesional cells was scored as negative ( $<5 \%$ of tumor cells), $1+$ (6-25\% of cells of tumor cells), $2+(26-50 \%$ of tumor cells) and $3+(>50 \%$ of tumor cells $)$.

P63 staining was considered positive by ascertaining only nuclear expression. Any cytoplasmic staining was considered background artifact. In each case, p63 was expressed in the normal epidermis in the nuclei of epidermal basal cells with a gradient i.e. a gradual decrease in expression of p63 in the more differentiated or superficial epidermal layers. Staining of the germinative layer of mature sebaceous glands, germinative hair matrix cells and the external root sheath of the hair follicles served as the positive internal controls. Similar to CK15, p63 expression was evaluated in the lesion in comparison to adjacent normal epidermis in each of the categories. Positive expression of p63 in the nuclei of lesional cells was scored as $0 \quad<25 \%$ of positively-staining tumor cells), $1+(25-75 \%$ of positively-staining tumor cells), and $2+(>75 \%$ of positively-staining tumor cells).

\section{Statistical Analysis}

The statistical association of expression of CK15, CK19 and p63 were analyzed using the Fisher's exact test to determine whether there were differences of significance in expression of the different stem cell markers amongst the categories studied. For purposes of statistical analyses, for CK15 and p63, scores of $1+$ or $2+$ were considered positive while for CK19, scores of $1+$ or more were considered positive. A two-tailed $P$ value of less than 0.05 was considered to be statistically significant.

\section{Results}

The distributions of CK15, CK19 and p63 are summarized in Table 1 and representative staining profiles depicted in Figure 1.

\section{Cytokeratin 15}

Overall immunoreactivity was observed in cases of actinic keratosis, squamous cell carcinoma in situ, bowenoid papulosis, and squamous cell carcinoma in a basilar pattern, defined by staining of the basal cell layer with or without scattered staining of the more differentiated keratinocytes (observed in only 3 of 30 cases of squamous cell carcinoma in situ).

In cases of actinic keratosis, retained immunoreactivity to CK15 was observed in 11/29 (38\%) ( 7 cases $1+$ and 4 cases $2+$ ), while in 18/29 cases (62\%) CK15 expression was lost; in cases of squamous cell carcinoma in situ, retained immunoreactivity to CK15 was observed in 16/30 cases (53\%) (7 cases $1+$ and 9 cases $2+$ ), while in 14/30 cases (47\%) CK15 expression was lost; in cases of bowenoid papulosis, 9/15 cases (60\%) showed immunoreactivity to CK15 (60\%) (6 cases $1+$ and 3 cases $2+$ ), while in 6/15 cases (40\%) CK15 expression was lost; in cases of squamous cell carcinoma, retained immunoreactivity to CK15 was observed in $1 / 29(3 \%)$ ( 1 case $2+$ ), while in $28 / 29$ cases (97\%) CK15 expression was lost.

Significant differences in expression of CK15 were noted between actinic keratosis and squamous cell carcinoma $(P<0.05)$, squamous cell carcinoma in situ and squamous cell carcinoma $(P<0.05)$, and bowenoid papulosis and squamous cell carcinoma $(P<0.05)$. Differences between actinic keratosis, squamous cell carcinoma in situ and bowenoid papulosis did not reach statistical significance.

\section{Cytokeratin 19}

Overall, immunoreactivity was patchy and predominantly in the basal epidermal layer in cases of actinic keratosis (4/6 cases), patchy and predominantly suprabasal in cases of squamous cell carcinoma in situ (10/11 cases) and bowenoid papulosis (1/2 cases) and, patchy suprabasal and, albeit rarely, diffuse in squamous cell carcinoma (6/7 cases) (Figure 2).

In cases of actinic keratosis, 6/29 cases (21\%) showed immunoreactivity to CK19 (3 cases $1+$ and

Table 1 Summary of CK15, CK19, and p63 expression in actinic keratosis, squamous cell carcinoma in situ, bowenoid papulosis, and squamous cell carcinoma

\begin{tabular}{|c|c|c|c|c|c|c|c|c|c|c|}
\hline & \multicolumn{3}{|c|}{ CK15 } & \multicolumn{4}{|c|}{ CK19 } & \multicolumn{3}{|c|}{ p63 } \\
\hline & $0 \%(\#)$ & $1+\%(\#)$ & $2+\%(\#)$ & $0 \%(\#)$ & $1+\%(\#)$ & $2+\%(\#)$ & $3+\%(\#)$ & $0 \%(\#)$ & $1+\%(\#)$ & $2+\%(\#)$ \\
\hline Actinic keratosis & $62(18 / 29)$ & $24(7 / 29)^{\mathrm{a}}$ & $14(4 / 29)^{\mathrm{a}}$ & $79(23 / 29)$ & $10(3 / 29)$ & $10(3 / 29)$ & $0(0 / 29)$ & $10(3 / 29)$ & $14(4 / 29)$ & $76(22 / 29)$ \\
\hline Squamous cell carcinoma in situ & $47(14 / 30)$ & $23(7 / 30)^{\mathrm{a}}$ & $30(9 / 30)^{\mathrm{a}}$ & $63(19 / 30)$ & $13(4 / 30)$ & $17(5 / 30)$ & $7(2 / 30)$ & $13(4 / 30)$ & $20(6 / 30)$ & $67(20 / 30)$ \\
\hline Bowenoid papulosis & $40(6 / 15)$ & $40(6 / 15)^{\mathrm{a}}$ & $20(3 / 15)^{\mathrm{a}}$ & $87(13 / 15)$ & $6.5(1 / 15)$ & $0(0 / 15)$ & $6.5(1 / 15)$ & $40(6 / 15)$ & $47(7 / 15)$ & $13(2 / 15)$ \\
\hline Squamous cell carcinoma & $97(28 / 29)$ & $0(0 / 29)^{\mathrm{a}}$ & $3(1 / 29)^{\mathrm{a}}$ & $76(22 / 29)$ & $17(5 / 29)$ & $0(0 / 29)$ & $7(0 / 29)$ & $0(0 / 29)$ & $0(0 / 29)$ & $100(29 / 29)$ \\
\hline
\end{tabular}

${ }^{\text {a }} P<0.05$ (actinic keratosis vs squamous cell carcinoma; squamous cell carcinoma in situ vs squamous cell carcinoma and bowenoid papulosis vs squamous cell carcinoma). 

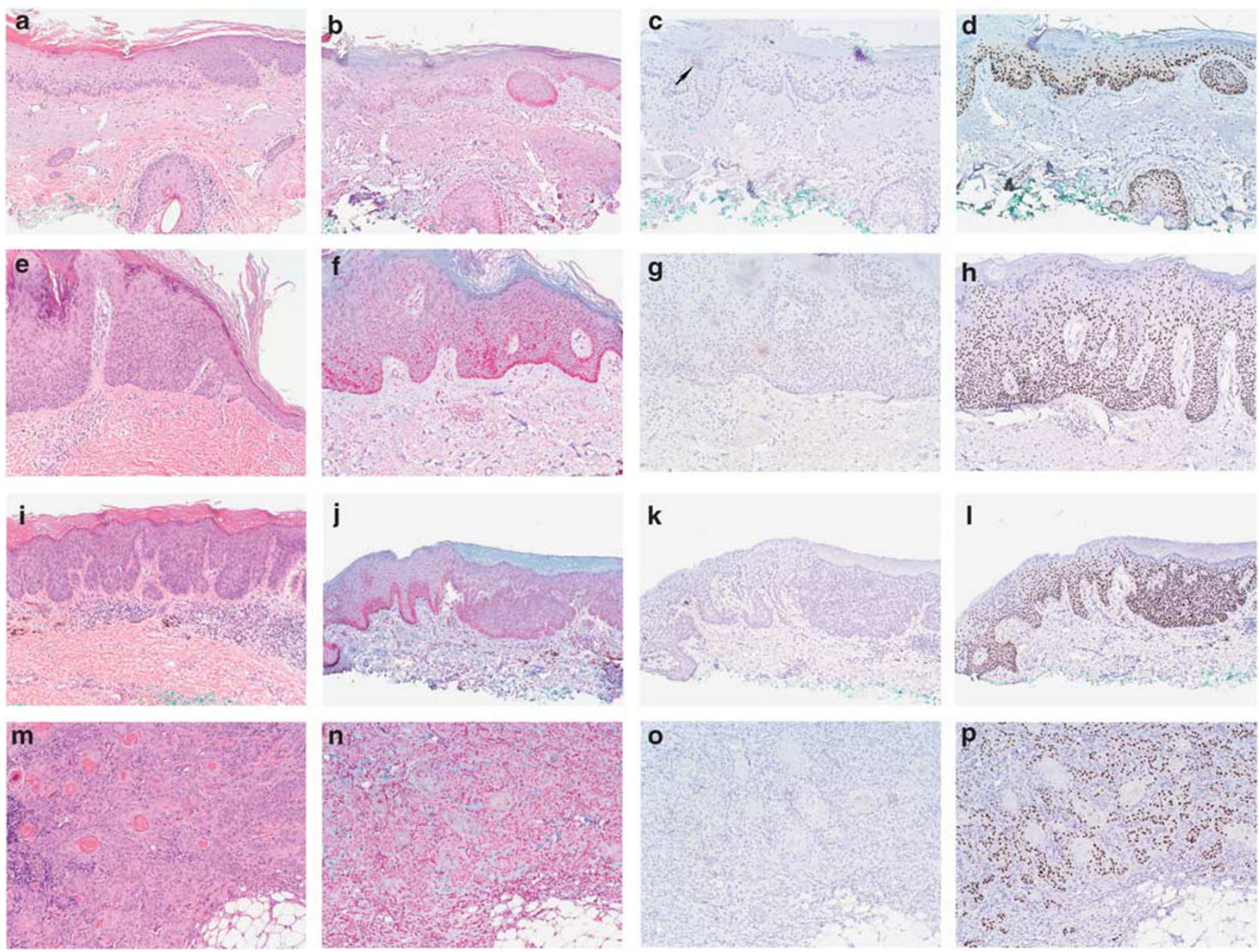

Figure 1 Representative examples of immunohistochemical staining profiles with stem cell markers CK15, CK19 and p63. (a-d) Actinic keratosis with loss of CK15 and retention of p63 (a) H\&E $10 \times$, (b) CK15, (c) CK19 and (d) p63. Arrow in c highlights positive staining of internal control i.e. eccrine coil. (e-h) Squamous cell carcinoma in situ with retention of CK15 and p63 (e) H\&E 10×, (f) CK15, (g) CK19 and (h) p63. (i-l) Bowenoid papulosis with retention of CK15 and p63 (i) H\&E $10 \times$, (j) CK15, (k) CK19 and (l) p63. (m-p) Squamous cell carcinoma, well differentiated with loss of CK15 and retention of p63 (m) H\&E $10 \times$ respectively, (n) CK15, (o) CK19 and (p) p63.

3 cases $2+$ ); in cases of squamous cell carcinoma in situ, $11 / 30$ cases $(37 \%)$ showed immunoreactivity to CK19 ( 4 cases $1+, 5$ cases $2+$, and 2 cases $3+$ ); in cases of bowenoid papulosis, $2 / 15$ cases $(13 \%)$ showed immunoreactivity to CK19 (1 case $1+$ and 1 case $3+$ ); in cases of squamous cell carcinoma, $7 / 29$ cases $(24 \%)$ showed immunoreactivity to CK19 ( 5 cases $1+$ and 2 cases $3+$ ) (Figure 2).

Differences between the groups did not reach statistical significance.

\section{p63}

Expression of p63 in cases of actinic keratosis and squamous cell carcinoma in which it was retained appeared comparable to that of the normal epidermis. A gradation in staining intensity was also observed in the latter group in the keratin pearls. In cases of squamous cell carcinoma in situ and bowenoid papulosis, uniform expression of p63 was noted in all epidermal layers.
In cases of actinic keratosis, 26/29 cases (90\%) showed immunoreactivity to p63 (4 cases $1+$, and 22 cases $2+$ ), while $3 / 29$ cases $(10 \%)$ cases exhibited loss of p63 expression; in cases of squamous cell carcinoma in situ, 26/30 cases (87\%) showed immunoreactivity to p63 (6 cases $1+$ and 20 cases $2+$ ), while $4 / 30$ cases (13\%) exhibited loss of p63 expression; in cases of bowenoid papulosis, 9/15 cases (60\%) showed immunoreactivity to p63 ( 7 cases $1+$ and 2 cases $2+$ ), while $6 / 15$ cases $(40 \%)$ exhibited loss of p63 expression; in cases of squamous cell carcinoma, 29/29 (100\%) retained p63 expression (all 29 cases $2+$ ).

Differences between the groups did not reach statistical significance.

\section{Discussion}

Cytokeratin 15, targeting keratinocytes in the bulge region, highlights a population of cells in follicular bulge with characteristics of stem cells. ${ }^{7}$ These cells 

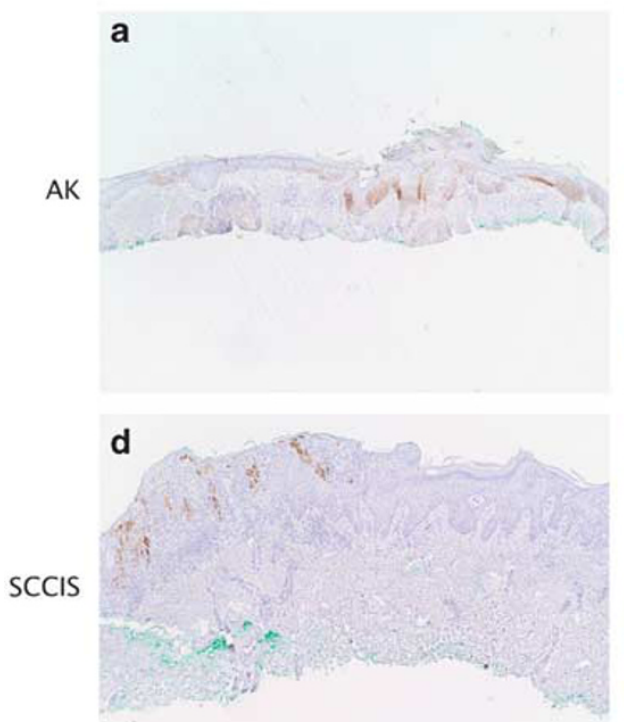

g

BP

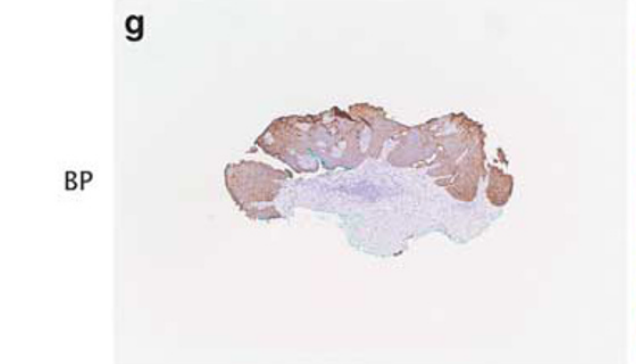

b
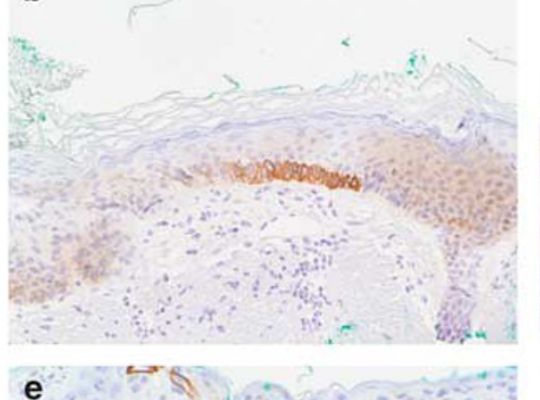

c

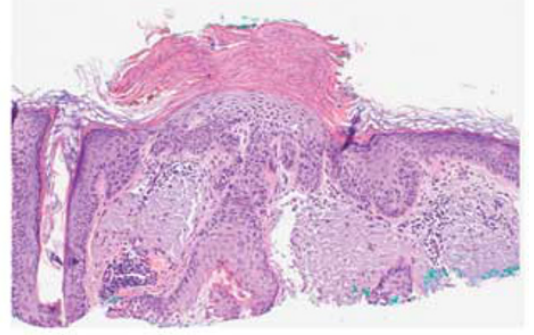

\section{f}

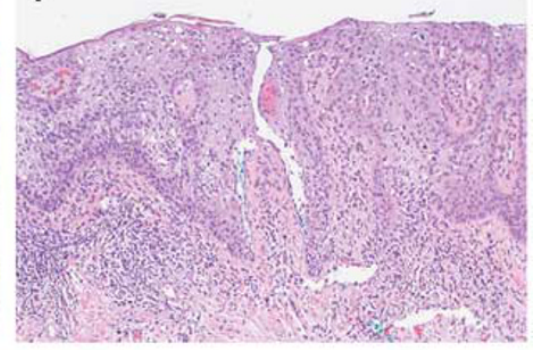

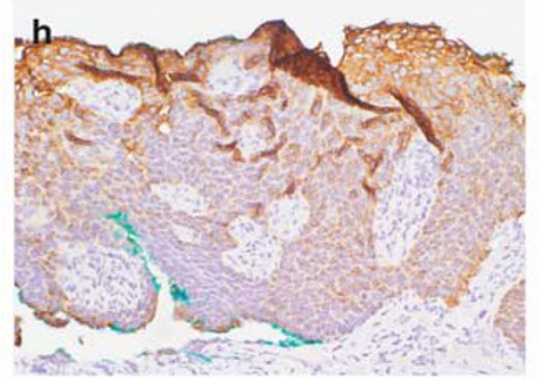
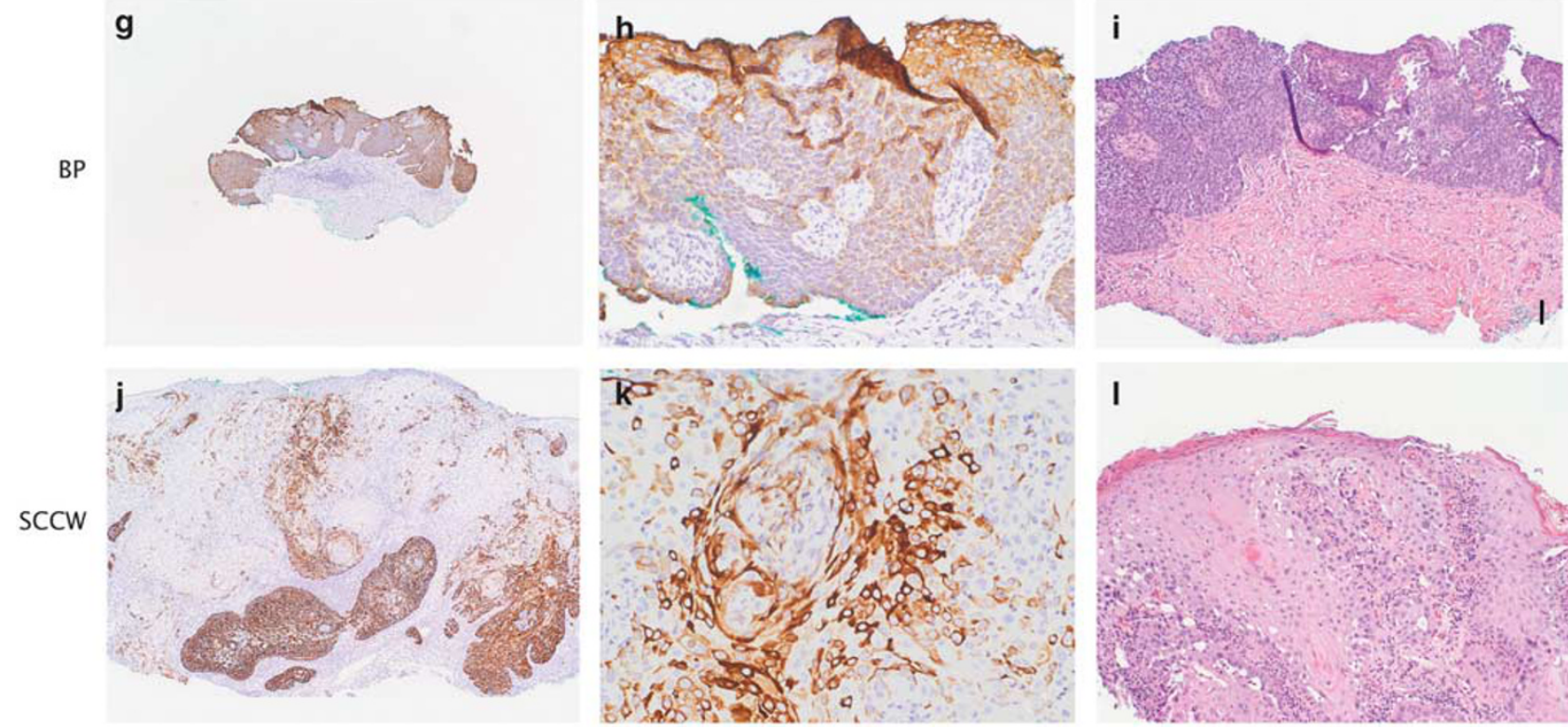

\section{I}

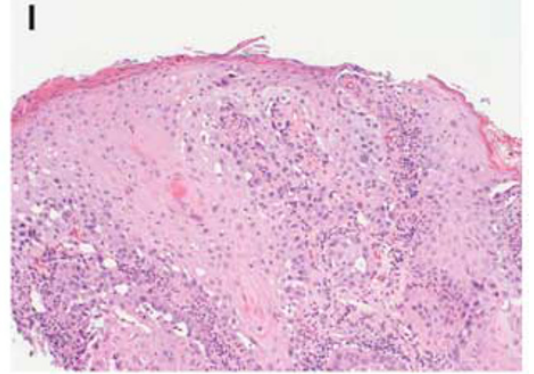

Figure 2 Representative examples of differing immunohistochemical staining with stem cell marker CK19. (a-c) Actinic keratosis with patchy and basal CK19 staining (a) $4 \times$, (b) $20 \times$, (c) H\&E $10 \times$. (d-f) Squamous cell carcinoma in situ with patchy and suprabasal CK19 staining (d) $4 \times$, (e) $20 \times$, (f) H\&E $10 \times$. (g-i) Bowenoid papulosis with patchy and suprabasal CK19 staining (g) $4 \times$, (h) $20 \times$, (i) H\&E $10 \times$. (j-l) Well-differentiated squamous cell carcinoma with patchy, suprabasal and diffuse CK19 staining (j) $4 \times$, (k) $20 \times$, (l) $\mathrm{H} \& \mathrm{E} 10 \times$.

appear to be not merely confined to the bulge region but have been identified in fetal and adult sebaceous glands as well as a spectrum of benign and malignant cutaneous neoplasms. ${ }^{7,16,17}$ Based on expression of CK15 in tumors with follicular differentiation, it is believed to be a relatively specific marker for neoplasms of putative hair follicle origin. In keeping with this hypothesis, we have previously reported expression of CK15 in microcystic adnexal carcinoma and desmoplastic trichepithelioma, with negative expression in squamous cell carcinoma including squamous cell carcinoma with ductal differentiation. ${ }^{16,18}$ Findings from the current study confirm this in that we found loss of CK15 in 97\% of cases of squamous cell carcinoma. Of interest, we noted that a significant number of CK15 positive stem cells were retained in atypical in situ epithelial lesions such as actinic keratosis (38\%), squamous cell carcinoma in situ $(53 \%)$ and bowenoid papulosis $(60 \%)$. What does this mean? A universally accepted paradigm is that to attain the complete transformed phenotype, a cell 
must accumulate multiple 'hits' in the form of progressive alterations within its chromosomes and irreversible changes in a number of genes. ${ }^{19} \mathrm{~A}$ stem cell with its lifespan comparable to the organism and inherent characteristic of 'slow cycling' is the perfect candidate for accumulations of such hits. ${ }^{20-22}$ Evidence for the role of stem cells in cancers is continually mounting with cells with stem cell-like features being identified in several malignancies. ${ }^{23-25}$ Examples include identification of leukemia cell lines with stem cell-like features, breast cancer stem cells and glial tumor stem cells with the capacity of differentiating into both glial and neuronal cell forms. ${ }^{26-28}$ Based on CK15 expression, findings from the current study expand the spectrum of lesions incriminating stem cells to include atypical in situ epithelial subgroups such as actinic keratosis, squamous cell carcinoma in situ and bowenoid papulosis. Retained CK15 expression in a sizable proportion of these lesions argues in favor of their origin from CK15-positive cells in the basal layer of the interfollicular epidermis. Loss of CK15 expression in squamous cell carcinoma suggests that it may be of relevance in the transformation of an in situ malignancy into one that is frankly invasive although additional studies are required to confirm this.

Aberrant expression of CK19 has been reported in oral and, more recently, in cutaneous squamous cell carcinoma and pagetoid squamous cell carcinoma in situ. ${ }^{29-31}$ In oral squamous cell carcinoma, CK19 expression correlated significantly with pathologic grade of differentiation in a study using immunohistochemistry and RT-PCR for CK19 mRNA detection. ${ }^{29}$ Similar to this, in cutaneous squamous cell carcinoma, Chen et al noted a significant increased expression of CK19 in cutaneous squamous cell carcinoma compared to actinic keratoses and Bowen's disease and, significant differences were also noted between CK19 positive populations harbored in Bowen's disease and actinic keratosis with higher numbers of CK19 positive cells noted in the former. ${ }^{32}$ In contrast, we observed no significant differences in CK19 expression between actinic keratosis $(21 \%)$, squamous cell carcinoma in situ $(37 \%)$, bowenoid papulosis $(13 \%)$, and squamous cell carcinoma $(24 \%)$. While a simplistic explanation is that the results are reflective of differences relating to clone of antibody or dilutions utilized, both studies utilized the same clone of antibody and similar dilutions (in both studies dilution was optimized to 1:100). However, the scoring system incorporated by us appears to be different from the one utilized by Chen et al with different cut-offs. ${ }^{32}$ In the study by Chen et al, staining of even $1 \%$ was considered positive in the series, while we considered $>6 \%$ to be positive. On a minor note, Chen et al also found a correlation between CK19 expression and the Ki-67 labeling index-a finding that is difficult to reconcile in light of the fact that stem cells are typically quiescent 'label retaining cells' while Ki-67 is a nuclear matrix protein that is expressed only in cycling cells not in cells that are resting. ${ }^{32}$ Of interest, in the 26 cases that demonstrated CK19 expression in our study, concurrent loss of CK15 was observed in $13(50 \%$; $2 / 6$ cases of actinic keratosis, $5 / 10$ cases of squamous cell carcinoma in situ and 6/7 cases of squamous cell carcinoma). While these findings imply a trend, differences between the groups of CK15-/CK19+ cells did not achieve statistical significance. We also noted differences in patterns of CK19 expression: in actinic keratosis the staining pattern was patchy and basal whereas in squamous cell carcinoma in situ, bowenoid papulosis and squamous cell carcinoma it was patchy and suprabasal. While it is unclear why CK19 positive stem cells 'home' to select portions of the epidermis, findings from the current study suggest that it does indeed happen. Although differences did not reach statistical significance, we also noted that a lower proportion of cases of bowenoid papulosis exhibited CK19 positivity compared to cases of squamous cell carcinoma in situ (13\% versus $37 \%$ ). Endocervical reserve cells, the putative target of HPV infection, have been shown to express p63 and CK17, both putatively identifying cervical stem cells. ${ }^{33}$ Findings from the current study imply that tentative HPV targets might include CK19 positive stem cells. Obviously additional studies such as ascertaining HPV status by in situ hybridization studies on microdissected populations of CK19 positive cells are required to provide conclusive and definitive evidence of the same.

P63, a nuclear transcription factor that triggers keratinocyte differentiation, is down-regulated in terminally differentiated cells. ${ }^{14,34,35}$ In previous studies, p63 expression has been noted in epidermal and adnexal basal/myoepithelial cells. ${ }^{36,37}$ Several studies have shown an increased expression of p63 in cutaneous squamous cell carcinoma, especially in the undifferentiated component of the tumors, suggesting a role for p63 in maintenance of the undifferentiated state of the tumor cells. ${ }^{14,38,39}$ Similar to previous studies, we observed p63 expression in all cases of squamous cell carcinoma but also noted expression in most cases of actinic keratosis $(90 \%)$, squamous cell carcinoma in situ $(87 \%)$, and bowenoid papulosis cases $(60 \%)$, with no statistically significant differences observed between different subgroups.

In sum, we confirm that there appears to be a significant presence of stem or progenitor cells in cutaneous in situ and invasive epithelial malignancies, reaffirming the theory that the epidermis contains hundreds of clonogenic keratinocytes. ${ }^{8,40}$ Differential localization of these three populations of stem cells argues in favor of stem cell heterogeneity, a feature already confirmed in follicular stem cells both by us and others. ${ }^{1,8}$ The profound cellular heterogeneity also raises the possibility that cells within these different compartments most 

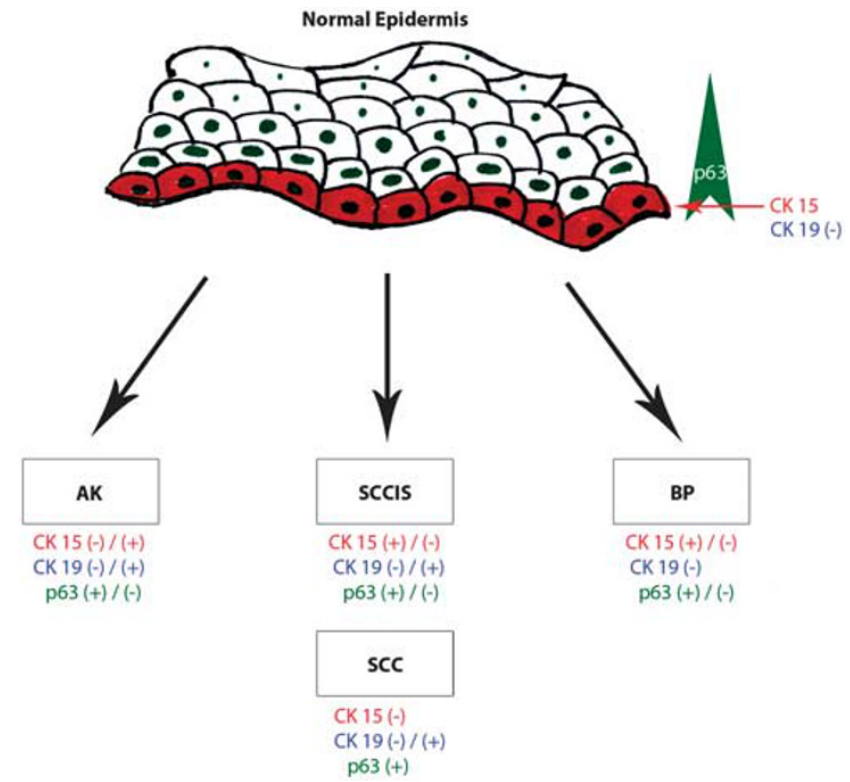

Figure 3 Schematic representation of stem cell marker expression most commonly observed in in situ and invasive malignancies based on findings from current study.

likely mirror individual developmental pathways in acquisition of the malignant phenotype, which is perhaps why only select stem cells play a role in the grading and development of in situ versus invasive malignancies (Figure 3).

\section{Disclosure/conflict of interest}

The authors declare no conflict of interest.

\section{References}

1 Abbas O, Mahalingam M. Epidermal stem cells: practical perspectives and potential uses. Br J Dermatol 2009;161:228-236.

2 Kloepper JE, Tiede S, Brinckmann J, et al. Immunophenotyping of the human bulge region: the quest to define useful in situ markers for human epithelial hair follicle stem cells and their niche. Exp Dermatol 2008;17:592-609.

3 Kamstrup MR, Gniadecki R, Skovgaard GL. Putative cancer stem cells in cutaneous malignancies. Exp Dermatol 2007;16:297-301.

4 Klein WM, Wu BP, Zhao S, et al. Increased expression of stem cell markers in malignant melanoma. Mod Pathol 2007;20:102-107.

5 Kanoh M, Amoh Y, Sato Y, et al. Expression of the hair stem cell-specific marker nestin in epidermal and follicular tumors. Eur J Dermatol 2008;18:518-523.

6 Braun KM, Niemann C, Jensen UB, et al. Manipulation of stem cell proliferation and lineage commitment: visualisation of label-retaining cells in whole mounts of mouse epidermis. Development 2003;130: 5241-5255.

7 Lyle S, Christofidou-Solomidou M, Liu Y, et al. The C8 144B monoclonal antibody recognizes cytokeratin 15 and defines the location of human hair follicle stem cells. J Cell Sci 1998;111:3179-3188.

8 Hoang MP, Keady M, Mahalingam M. Stem cell markers (cytokeratin 15, CD34 and nestin) in primary scarring and nonscarring alopecia. Br J Dermatol 2009; 160:609-615.

9 Pozdnyakova O, Mahalingam M. Involvement of the bulge region in primary scarring alopecia. J Cutan Pathol 2008;35:922-925.

10 Lyle S, Christofidou-Solomidou M, Liu Y, et al. Human hair follicle bulge cells are biochemically distinct and possess an epithelial stem cell phenotype. J Investig Dermatol Symp Proc 1999;4:296-301.

11 Michel M, Török N, Godbout MJ, et al. Keratin 19 as a biochemical marker of skin stem cells in vivo and in vitro: keratin 19 expressing cells are differentially localized in function of anatomic sites, and their number varies with donor age and culture stage. J Cell Sci 1996;109:1017-1028.

12 Morris RJ. A perspective on keratinocyte stem cells as targets for skin carcinogenesis. Differentiation 2004;72: 381-386.

13 Pellegrini G, Dellambra E, Golisano O, et al. p63 identifies keratinocyte stem cells. Proc Natl Acad Sci USA 2001;98:3156-3161.

14 Reis-Filho JS, Torio B, Albergaria A, et al. p63 expression in normal skin and usual cutaneous carcinomas. J Cutan Pathol 2002;29:517-523.

15 Koster MI, Kim S, Roop DR. p63 deficiency: a failure of lineage commitment or stem cell maintenance? J Investig Dermatol Symp Proc 2005;10:118-123.

16 Jih DM, Lyle S, Elenitsas R, et al. Cytokeratin 15 expression in trichoepitheliomas and a subset of basal cell carcinomas suggests they originate from hair follicle stem cells. J Cutan Pathol 1999;26:113-118.

17 Bieniek R, Lazar AJ, Photopoulos C, et al. Sebaceous tumours contain a subpopulation of cells expressing the keratin 15 stem cell marker. Br J Dermatol 2007;156:378-380.

18 Hoang MP, Dresser KA, Kapur P, et al. Microcystic adnexal carcinoma: an immunohistochemical reappraisal. Mod Pathol 2008;21:178-185.

19 Knudson AG. Hereditary cancer: two hits revisited. J Cancer Res Clin Oncol 1996;122:135-140.

20 Tiede S, Kloepper JE, Bodo E, et al. Hair follicle stem cells: walking the maze. Eur J Cell Biol 2007;86: 355-376.

$21 \mathrm{Li} \mathrm{L}$, Xie T. Stem cell niche: structure and function. Annu Rev Cell Dev Biol 2005;21:605-631.

22 Lechler T, Fuchs E. Asymmetric cell divisions promote stratification and differentiation of mammalian skin. Nature 2005;437:275-280.

23 Sirard C, Lapidot T, Vormoor J, et al. Normal and leukemic SCID-repopulating cells (SRC) coexist in the bone marrow and peripheral blood from CML patients in chronic phase, whereas leukemic SRC are detected in blast crisis. Blood 1996;87:1539-1548.

24 Cobaleda C, Gutiérrez-Cianca N, Pérez-Losada J, et al. A primitive hematopoietic cell is the target for the leukemic transformation in human philadelphia-positive acute lymphoblastic leukemia. Blood 2000;95: 1007-1013.

25 Singh SK, Clarke ID, Terasaki M, et al. Identification of a cancer stem cell in human brain tumors. Cancer Res 2003;63:5821-5828. 
26 Morrison SJ, Weissman IL. The long-term repopulating subset of hematopoietic stem cells is deterministic and isolatable by phenotype. Immunity 1994;1: 661-673.

27 Oliveira LR, Jeffrey SS, Ribeiro-Silva A. Stem cells in human breast cancer. Histol Histopathol 2010;25: 371-385.

28 Park DM, Rich JN. Biology of glioma cancer stem cells. Mol Cells 2009;28:7-12.

29 Zhong LP, Chen WT, Zhang CP, et al. Increased CK19 expression correlated with pathologic differentiation grade and prognosis in oral squamous cell carcinoma patients. Oral Surg Oral Med Oral Pathol Oral Radiol Endod 2007;104:377-384.

30 Takeda T, Sugihara K, Hirayama Y, et al. Immunohistological evaluation of Ki-67, p63, CK19 and p53 expression in oral epithelial dysplasias. J Oral Pathol Med 2006;35:369-375.

31 Raju RR, Goldblum JR, Hart WR. Pagetoid squamous cell carcinoma in situ (pagetoid Bowen's disease) of the external genitalia. Int J Gynecol Pathol 2003;22: 127-135.

32 Chen S, Takahara M, Kido M, et al. Increased expression of an epidermal stem cell marker, cytokeratin 19, in cutaneous squamous cell carcinoma. Br J Dermatol 2008;159:952-955.
33 Martens JE, Arends J, Van der Linden PJ, et al. Cytokeratin 17 and p63 are markers of the HPV target cell, the cervical stem cell. Anticancer Res 2004;24: 771-775.

34 Levrero M, De Laurenzi V, Costanzo A, et al. The p53/ p63/p73 family of transcription factors: overlapping and distinct functions. J Cell Sci 2000;113:1661-1670.

35 Pellegrini G, Dellambra E, Golisano O, et al. p63 identifies keratinocyte stem cells. Proc Natl Acad Sci USA 2001;98:3156-3161.

36 Reis-Filho JS, Schmitt FC. Taking advantage of basic research: p63 is a reliable myoepithelial and stem cell marker. Adv Anat Pathol 2002;9:280-289.

37 Tsujita-Kyutoku M, Kiuchi K, Danbara N, et al. p63 expression in normal human epidermis and epidermal appendages and their tumors. J Cutan Pathol 2003;30: 11-17.

38 Wrone DA, Yoo S, Chipps LK, Moy RL. The expression of p63 in actinic keratoses, seborrheic keratosis, and cutaneous squamous cell carcinomas. Dermatol Surg 2004;30:1299-1302.

39 Dotto JE, Glusac EJ. p63 is a useful marker for cutaneous spindle cell squamous cell carcinoma. J Cutan Pathol 2006;33:413-417.

40 Hoffman RM. The pluripotency of hair follicle stem cells. Cell Cycle 2006;5:232-233. 